\title{
El impacto de ley 1696 del 2013 sobre la demanda y consumo de alcohol etílico. Caso: aguardiente blanco del Valle de la ILV
}

\author{
The impact of law 1696 of 2013 on the demand and consumption of ethyl alcohol. \\ Case: aguardiente blanco del valle de la ILV
}

\section{Resumen}

Este trabajo trata del impacto sobre la demanda por alcohol etílico (botella de aguardiente blanco del Valle 750 cc), en el marco de la Ley 1696 de 2013, el caso del departamento del Valle del Cauca. Se comprende que la legislación en Colombia ha hecho un esfuerzo importante para regular, no solo la venta, sino los efectos nocivos que el alcohol pueda tener sobre los consumidores y la sociedad (Rehm, et al; 2009). Los estudios muestran que Colombia es un importante consumidor de bebidas alcohólicas a nivel continental y que el consumo del mismo es muy representativo dentro del gasto de consumo del ciudadano medio. A través de un de un modelo ARIMA, se ha logrado detectar que no hay evidencia significativa para indicar que el la Ley 1696 del 2013 tenga un efecto regresivo sobre la demanda del producto en estudio. Se infiere que, si bien la ley no busca desestimular el consumo de alcohol, sí se esperaba que la ley tuviese un efecto "indirecto" sobre el consumo. Implícitamente, el estudio infiere que la demanda de alcohol no está correlacionada con quienes tienen vehículos y conducen, sino que la misma está asociada a estrategias propias de ese producto en el mercado.

Palabras Clave: Bebidas alcohólicas, modelos ARIMA, Ley 1696 2013, determinantes de demanda, consumo de alcohol.

\section{Resumo:}

Este trabalho trata do impacto sobre a demanda de álcool etílico (garrafa de aguardente blanco del valle 750 cc), no âmbito da lei 1.696 de 2013, no caso do departamento Valle del Cauca. Se compreende que a legislação na Colômbia fez um esforço importante para regular, não somente a venda, como também os efeitos nocivos que o álcool pode ter sobre os consumidores e a sociedade. Os estudos mostram que

* Economista. Magister Ingeniería Industrial. Universidad del Valle. Grupo de Investigación Interdisciplinario en Conocimiento Organizacional. Fundación Universitaria Católica Lumen Gentium.dfpena@unicatolica.edu.co 
a Colômbia é um importante consumidor de bebidas alcoólicas a nível continental, e que o consumo do mesmo é muito representativo dentro do gasto médio de consumo do cidadão. Através do modelo "ARIMA", foi possível detectar que não há evidências significativas para indicar que a lei 1.696 de 2013 tenha um efeito regressivo sobre a demanda do produto em estudo. Infere-se que a lei não busca desestimular o consumo de álcool, embora se esperasse que a lei tivesse um efeito "indireto" sobre o consumo. No implícito estudo infere-se que a demanda de álcool não esta correlacionada com quem tem veículos e conduz mas sim à estratégias próprias desse produto no mercado

Palavras Chave: Bebidas alcoólicas, modelos ARIMA, Ley 1696 2013, determinantes da demanda, consumo de álcool.

\section{Introducción}

El consumo de bebidas alcohólicas en el país está determinado por las preferencias de consumo según regiones; en la región Cundinamarca y Boyacá predomina la cerveza; en el eje cafetero, el aguardiente; y en la costa norte, el whisky y el ron. Colombia tiene un consumo de alcohol de 6.3 litros por habitante; de los cuales, el $66 \%$ es cerveza y el $33 \%$ otros licores. En términos de producción, la industria cervecera representa cerca del $83 \%$, siendo la producción de alcohol no mayor al $5 \%$. La tendencia en el consumo muestra que la ingesta de cerveza creció a una tasa del $7.3 \%$ anual entre el 2003-2010. En el mismo periodo, el consumo del vino lo hacía al $14.3 \%$ y el aguardiente al $2.1 \%$. (Zapata y Sabogal, 2011).

Según la Organización Mundial de la Salud OMS (2002), el alcohol es el tercer factor de riesgo para la salud de los ciudadanos en los países desarrollados, a corta distancia del tabaco y la hipertensión arterial. En ese sentido, la legislación busca una regulación cada vez más drástica en términos de su vigilancia y control; entre la normatividad se desatacan en su or- den: la Ley 124 de 1994, que prohíbe la venta de licor a menores de edad y adopta medidas para menores que se encuentren en estado de beodez. También se tiene, el artículo 20 de la Ley 1098 de 2006, que reglamenta la protección de menores ante el consumo y el uso de estos en la comercialización de sustancias psicoactivas y alcohólicas. De igual forma, el artículo 47 de la Ley 1098 de 2006, prohíbe la publicidad de alcohol en horarios de franja infantil. En ese sentido, en el año 2010, se expide el decreto 120 del 2010, que busca la protección de menores y de la población en general de los efectos nocivos del consumo de alcohol y establece medidas tendientes a la reducción del daño y la minimización del riesgo de accidentalidad, violencia cotidiana y criminalidad asociada al consumo inmoderado de alcohol. En 2013, se expide la Ley 1696 de 2013, la cual tiene por objeto establecer sanciones penales y administrativas a la conducción de vehículos automotores bajo el influjo del alcohol u otras sustancias psicoactivas. Sin duda, la Ley 1696 de 2013 puede crear un incentivo negativo al consumo de bebidas alcohólicas. La Ley 1696 deja implícito de que exista una correlación positiva entre el uso de vehículos automotores, accidentes y alcohol. Es importante ver el impacto de esta ley sobre la demanda. Es factible también creer que el efecto de la ley no sea que las personas dejen de consumir bebidas alcohólicas, sino más bien la prevención del impacto del uso de vehículos automotores por conductores alicorados. En general, no todos los que consumen alcohol conducen, no todos los que conducen toman bebidas alcohólicas, es por ello importante ver si el impacto sobre la demanda es significativo, marginal o inocuo.

Puede haber otros efectos adversos, como en el caso de las rentas departamentales, toda vez que el consumo de alcohol es una fuente de ingresos departamentales, y el uso eficiente de la capacidad de planta de las licoreras que cada vez se ve afectada. Para el año 2014, de las licoreras existentes en el país, solo 6 licoreras departamentales arrojan aceptables resultados financieros, estas en su mayoría ya no producen alcohol y solo envasan aguardiente, ron y algunos aperitivos. La capacidad promedio instalada de una 
licorera está alrededor de 60 millones de botellas, pero solo se producen 15 que representa únicamente el $25 \%$. Este trabajo muestra el posible efecto sobre la demanda y el consumo de alcohol dado la Ley 1696 de 2013.

\section{Antecedentes}

Los estudios de demanda de alcohol en Colombia son relativamente pocos. Afanador y Joya (2013) describen que la demanda de bebidas espirituosas en Colombia es dinámico, creciente y competitivo; se indica que el país es actualmente el tercero de la región en consumo de bebidas alcohólicas, detrás de Venezuela y Brasil. Destacando que la cerveza es el producto de mayor consumo con el $90 \%$, seguida por el aguardiente y ron. Así mismo, se revela que los productos importados como whisky, vodka y vinos han ido ganando mercado, asociado a un incremento en el ingreso y a las preferencias arancelarias gracias a los acuerdos comerciales.

Manquillo, (Mora y Sánchez; 2012), indican que el precio como variable de demanda presenta características de variable no manipulada, es decir, no hace parte el control del oferente, debido a que los impuestos son constantes, muy altos y dependen del grado alcoholimétrico de la bebida. Los impuestos están establecidos por la Ley 788 del 2002, y se indexan de acuerdo al índice de precios al consumidor (IPC). En general, se muestra que para el sector de las bebidas alcohólicas en Colombia, el precio representa una variable clave al momento de decidir qué producto adquirir, ya que debido a la gran competencia de licores nacionales e importados, las empresas participan fuertemente por atraer más mercado y es precisamente el valor que le dan a sus productos un factor decisivo para los consumidores. También se señala que el mercado está regulado por barreras de entrada, además cada región tiene diferentes controles legales que impiden la entrada de competencia externa, en virtud a que mantienen la existencia de monopolios departamentales. También se indica que variables como la presentación física del producto es importante y es un determinador y señal básica para los consumidores en el momento de la elección. Se destaca que la segmentación es súper estudiada y que su tratamiento es científico, dirimir y decidir qué tipo de licor, a qué segmento, a qué sexo, a qué edad, a qué actividad e ingreso del consumidor es lo que determina el tipo de licor a colocar en ciertos nichos de mercado. Finalmente, se señala que otras variables sensibles del mercado son la publicidad, plaza y tecnología.

El ministerio de Salud y Protección Social Minsalud (2013), establece que, en cuanto al consumo de bebidas alcohólicas, el $87 \%$ de los encuestados declara haber consumido alguna vez en la vida, y el 35.8 $\%$ manifiesta haber consumido en los últimos 30 días (8,3 millones de personas). De igual forma, detalla el informe que se observan claras diferencias por sexo: mientras el $46.2 \%$ de los hombres manifiesta haber consumido alcohol en el último mes, entre las mujeres la cifra es del $25.9 \%$. En términos de la edad, la mayor tasa de uso actual de alcohol, del $49.2 \%$, se presenta entre los jóvenes de 18 a 24 años, y con un valor del $45.4 \%$ se define el consumo entre los jóvenes de 25 a 34 años. Le sigue el grupo de 35 a 44 años y luego los de 45 a 65 años. La diferencia entre estos es de 10 puntos de porcentaje. Los adolescentes de 12 a 17 años son quienes menor tasa de consumo presentan, del $19.3 \%$. El consumo actual de bebidas alcohólicas es mayor conforme aumenta el nivel socioeconómico de la población, pasando del 32 \% en el estrato más bajo, al $42 \%$ en el estrato más alto. También, el consumo de alcohol se vincula con el nivel de urbanización en el cual habitan las personas. En los centros más urbanizados como Bogotá, Medellín, Cali, Yumbo, Barranquilla y Soledad, las prevalencias de consumo en el último mes alcanzan al $38 \%$ y 39 \% de la población.

Las leyes contra la lucha antialcohólica datan de principios del siglo 20, de acuerdo con Garzón (2011) la década de los años veinte (20) en Colombia, para muchos historiadores, fue el inicio del proceso de modernización de una sociedad con fuertes atrasos en lo político, lo económico, social y cultural. En este 
ambiente de cambios, se promulgó una legislación acerca de licores que señaló el futuro tanto de la producción como del consumo de estas bebidas a nivel nacional. Esta legislación facultó a los gobernadores para la eliminación de las rentas de licores en los departamentos, buscando el control en la producción, comercialización y consumo.

Sierra (2011) muestra que en Colombia se ha buscado desincentivar el consumo de alcohol, a través de mecanismos legales. Las dos leyes de la lucha antialcohólica -la Ley 88 de 1923 y la Ley 88 de 1928 respectivamente- fueron los árbitros de la política fiscal de licores para la primera mitad del siglo XX. Se indica que la aplicación de la legislación en la lucha antialcohólica en los departamentos no es homogénea, ya que medidas como la implantación del sistema de administración directa de la renta de licores se hizo de forma inmediata, a diferencia de la implantación del sistema de litraje para la renta de licores fermentados que tardó una década. Este mismo autor, pone de manifiesto que las medidas fiscales fueron novedosas para el estado colombiano y benefició directamente a los tesoros departamentales. Este carácter modernizador de las dos leyes en la lucha antialcohólica de los años 30 se basó, básicamente, en los cambios administrativos y fiscales ocasionados con esta legislación de licores. Es indispensable determinar que un siglo de existencia del Estado-Nación colombiano bastó para la reestructuración de la política fiscal de licores, la cual ejerció un mayor control sobre la producción, comercialización y consumo de todo tipo de licores, que antes era imposible de llevar a cabo.

Por otra parte, Zuleta (2000) en un estudio para Fedesarrollo, establece que las altas tasas de impuestos son, junto con la complejidad de su estructura, administración y aplicación, los elementos de mayor distorsión del mercado colombiano de licores. Llegando a la conclusión de que los impuestos excesivos tienden a reducir el mercado legal y a deprimir el consumo en general. Así mismo, estos impuestos excesivos acrecientan los incentivos de altas ganancias a los contrabandistas, los lavadores de dinero, los vendedores de licor adulterado y los exportadores de licores en forma ficticia. Finalmente, la Ley 1696 de 2013, por medio de la cual se dictan disposiciones penales y administrativas para sancionar la conducción bajo el influjo de alcohol u otras sustancias psicoactivas. Tenjo (2013), advierte que la mencionada ley, a pesar de su impacto sobre las muertes por accidentalidad (que es, sin duda, un aspecto muy importante), no tiene ningún efecto sobre problemas menos visibles pero que son tan o más importantes que la accidentalidad.

\section{Algunos aspectos teóricos}

El problema se observa desde diferentes referentes teóricos asociados a la demanda. La demanda, relaciona el comportamiento de un agente económico que en su carácter de consumidor de bienes y de servicios busca, a través de la combinación de estos y de acuerdo a sus preferencias y una restricción presupuestal, alcanzar un nivel máximo de utilidad.

Dentro de los postulados teóricos sobresale la teoría de la demanda neoclásica que plantea que las preferencias del consumidor pueden modelizarse como una función de utilidad convexa y al menos dos veces diferenciable; en donde la función de utilidad mide la utilidad de un consumidor cuando disfruta del consumo de un determinado bien, suponiendo que el consumidor es perfectamente racional y la convexidad hace alusión al conjunto de preferencias que el consumidor debe decidir y que optimiza la utilidad dada una restricción presupuestal. Dentro de la estructura neoclásica se destaca que la utilidad no varía en el proceso de consumo, esto es que el consumidor se comporta igual a si antes de consumir decidiera sobre cómo distribuir la renta disponible (en lugar de ajustar adaptativamente el consumo a medida que gasta la renta disponible). En el escenario planteado, dado la información disponible, un consumidor racional consumirá cantidades de cada bien, de manera que la utilidad total sea la máxima y compatible con su 
nivel de ingreso. En el marco de acción neoclásica, la consecuencia de la actuación racional del consumidor en la función de demanda, implica que no existen bienes insustituibles, violentando el principio de la inelasticidad de la demanda de algunos tipos de bienes que en realidad sí son insustituibles.

La teoría Pos keynesiana, admite que las preferencias de los consumidores tienen una estructura lexicográfica ${ }^{1}$ incompatible con la utilidad escalar. Así, el consumidor dividiría los bienes entre categorías y pre asignaría una parte de la renta a cada categoría, distribuyendo entre los bienes de cada una el presupuesto; en ese sentido, Georgescu-Roegen (1971) indican que, en general, se considera que los consumidores gastan su dinero de esta manera, creándose de esa forma un principio de saciedad ${ }^{2}$. De esta manera, la racionalidad en el sentido neoclásico es una falacia; la racionalidad no puede ser infinita. Según Simon (1954), la hipótesis básica de la economía neoclásica, en donde los agentes tienden a maximizar los resultados de sus comportamientos, es muy limitada, en la práctica ningún ser humano está continuamente buscando la solución óptima. Aunque deseara hacerlo, el coste de informarse sobre todas las alternativas y la incertidumbre sobre el futuro lo harían imposible. De acuerdo con Simón, las personas intentan buscar una mínima satisfacción, es decir, tratan de alcanzar ciertos niveles de éxito para después, poco a poco, ir ajustando esa solución. Esta estructura de pensamiento fue la que Simon denominó racionalidad limitada ${ }^{3}$.

En el argumento Pos Keynesiano también aparece el principio de la Separación de Lancaster (1971, citado por Lavoie, 2004, P. 39) en donde se dice que

\footnotetext{
1 Es un caso especial de preferencias del consumidor que pertenece, de hecho, a la Teoría del consumidor. El nombre proviene de la forma en que se organiza un diccionario, ya que, en este caso, se le da alta prioridad al ordenamiento de las preferencias de forma análoga a como se ordenan las palabras, por letras, en un diccionario.

2 El Principio de saciedad establece que más allá de un umbral finito la necesidad queda satisfecha y consumir más unidades no aumenta la satisfacción o "utilidad".

3 La racionalidad limitada se establece como la racionalidad procedimental, que se comprende cuando el consumidor se rige por reglas o hábitos no compensadores
}

el consumidor subdivide sus elecciones y necesidades en diversas categorías, débilmente relacionadas unas con otras. Eso implica que los cambios en los índices de precios de un tipo de productos asociados a determinadas necesidades, no afecta a las cantidades consumidas de otras categorías, ya que estas son básicamente independientes. Así, difícilmente una cantidad insuficiente de alimento puede ser compensada por una mayor cantidad de oferta cultural, dado que, probablemente, el alimento y el deseo de ocio pertenecen a categorías diferentes de deseos y necesidades. También, el Pos Keynesianismo, indica la existencia de la jerarquización de las necesidades, enfatizando que estas, a menudo, son subordinadas unas a otras. La distribución del presupuesto no consiste en maximizar una utilidad entre bienes disponibles, sino que los bienes situados en un nivel jerárquico no son consumidos, a menos que estén mínimamente satisfechas las necesidades de bienes de los niveles jerárquicos inferiores. Esto implica, en algunos casos, que el orden de preferencias de combinaciones de bienes siga un orden lexicográfico, si eso sucede, entonces, la función de utilidad de las preferencias asociada a la utilidad marginal tiene que estar representada por un vector, cuyos componentes estarían asociados a un determinado nivel jerárquico de necesidades (Martínez 2004).

También se establece que en la comprensión de la demanda y de las preferencias d e los consumidores se presenta el principio del crecimiento. Tratado por Georgescu-Roegen y Pasinetti (1971), establece que el tiempo y el crecimiento de la renta disponible para un consumidor hacen que sus preferencias evolucionen escalonadamente y el acceder a niveles de renta superiores hace que se consideren necesidades que previamente no habían sido consideradas. Galbraith (1941), trató que la demanda y las preferencias de los agentes estuvieran determinadas por lo que él denominó el principio de dependencia, el cual indica que el reconocimiento y la formación de los gustos dependen de la publicidad, las modas, el grupo social al que pertenece el agente económico y no simplemente de unos gustos autónomos objetivos. Finalmente, aparece el principio de herencia, este establece que las 
preferencias actuales de un consumidor dependen de su historia pasada de consumo. Las elecciones de hoy están condicionadas por las elecciones de ayer, esto hace que la dinámica de consumo, a lo largo del tiempo, no depende de maximizar una función de utilidad objetiva e inmutable, sino que nuestra historia de elecciones pasadas puede ser lo más determinante en la configuración de nuestros gustos actuales.

Entre otros enfoques está el cardinalista de la demanda, el cual implica que en las elecciones entre los diferentes bienes que obtiene un consumidor existe una utilidad que puede ser medida en utilidad total y la utilidad marginal. También existe el enfoque ordinalista donde se da importancia a la ordenación de las preferencias, sin que sea necesario que los individuos le asignen un valor numérico, lo importante es que una combinación de bienes proporciona la misma o menos utilidad que otra. Por este motivo, el concepto de utilidad aparece ligado a la visión cardinalista de las preferencias. Según este enfoque, la función de utilidad no ha de representar una magnitud ordinal o medible.

En términos de preferencias (Martínez, 2004), estas se definen como una relación ordinal entre distintos estados sobre los que el individuo puede elegir. Dada una alternativa entre dos elementos simples (un bien) o compuestos (una cesta de bienes), pudiendo generalizar los bienes a estados o situaciones, el individuo sabe cuál es el par de la comparación que prefiere. Para permitir una elección racional, deben cumplir tres supuestos que permiten una ordenación de las mismas: ser reflexivas, completas y transitivas:

Martínez (2004; citado por Cante, 2007, p. 12), y este citando a Frankfurt (1971) y Harsanyi (1955), relacionan los términos de Metapreferencias ${ }^{4}$ y prefe-

4 En realidad, las personas tienen deseos de segundo orden o Metapreferencias que no sólo incluyen a sus semejantes, también, dan un sentido a su vida (dan una dirección y un significado a sus escogencias cotidianas de primer orden). Desde esta perspectiva, el dilema del prisionero con el cual se asocia, simplistamente, el problema de la inacción colectiva, conduce a desastres colectivos cuando los individuos prefieren cuidar de sí mismos, pero si existen Metapreferencias (cierta moralidad, compromisos colectivos o algunas preferencias sociales), puede existir cooperación. (Cante, 2007). rencias éticas, para referirse a un problema similar, es decir, a un tipo de preferencias superior que ordena otro tipo de predilecciones. Por ejemplo, decidir una identidad, como ser monje de clausura, ordena mis preferencias sobre innumerables cuestiones. Pero esta estrategia explicativa plantea el problema de la regresión infinita, pues habría preferencias de tercer orden para elegir entre preferencias de segundo orden y así, sucesivamente.

\section{Aspectos legales}

El alcoholismo es una enfermedad social y por ende responsable de muchas de las difusiones de comportamiento social que se presentan (OMS, 2002) y en ese sentido, la legislación busca una regulación cada vez más drástica en términos de su vigilancia y control. Entre algunas normas, decretos y leyes se desatacan en su orden: Decreto 1355 de 1970, el cual establece y dicta normas sobre Policía, en el artículo 111, para que la policía fije zonas y horarios para el funcionamiento de establecimientos donde se expendan bebidas alcohólicas. Más adelante, la Ley 30 de 1986, por la cual se adopta el Estatuto Nacional de Estupefacientes y se dictan otras disposiciones, en el capítulo 3, se establecen campañas de prevención contra el consumo del alcohol y del tabaco. Una de las leyes más importante es la Ley 124 de 1994, la cual prohíbe la venta de licores a menores de edad y adopta medidas para el manejo de menores que se encuentren en estado de beodez. La misma, se complementó con el artículo 20 de la Ley 1098 de 2006, que reglamenta la protección de menores ante el consumo y el uso de estos en la comercialización de sustancias psicoactivas y alcohólicas. De igual forma, el artículo 47 de la Ley 1098 de 2006, que prohíbe la publicidad de alcohol en horarios de franja infantil. En ese sentido, se da el decreto 120 del 2010, que busca la protección de menores y de la población en general de los efectos nocivos del consumo de alcohol y establece medidas tendientes a la reducción del daño y la minimización del riesgo de accidentalidad, vio- 
lencia cotidiana y criminalidad asociada al consumo inmoderado de alcohol.

En el Decreto 120 de 2010, se adoptan medidas en relación con el consumo de alcohol. El artículo 23, establece que los distritos y municipios promoverán la realización de Pactos por la Vida entre los residentes de las zonas consideradas como críticas, los dueños de establecimientos comerciales donde se expenda y se consuma alcohol y los centros educativos del área de influencia en las zonas identificadas como críticas, con los que se definan estrategias orientadas a la disminución del daño y la minimización del riesgo que evite los desenlaces de accidentes, violencia cotidiana y criminalidad que acompañan los ambientes de consumos abusivos. Dichos pactos deberán contener medidas especiales de protección al menor de edad frente al consumo de alcohol y sus consecuencias. En 2013, se expide la Ley 1696, la cual tiene por objeto establecer sanciones penales y administrativas a la conducción bajo el influjo del alcohol $\mathrm{u}$ otras sustancias psicoactivas.

En Colombia, los accidentes de tránsito provocados por conductores en estado de embriaguez han sido algo cotidiano en el transcurso de los años, los cuales no habían tenido el rigor jurídico que pudiera contrarrestarlos. En la actualidad, se han desarrollado un sin número de proyectos de ley que buscan castigar estas conductas. La ley 1696 de 2013 que rige a nivel nacional, por medio de la cual se dictan disposiciones penales y administrativas para sancionar la conducción bajo el influjo del alcohol $u$ otras sustancias psicoactivas.

\section{Aspectos metodológicos}

El objetivo del presente trabajo es evidenciar el efecto de la Ley 1696 del 19 de diciembre de 2013 que dicta disposiciones penales y administrativas para sancionar la conducción bajo el influjo del alcohol $u$ otras sustancias psicoactivas, sobre las unidades consumidas de alcohol. En este caso, en el departamento del Valle sobre el producto Aguardiente Blanco del Valle.

Metodológicamente se utilizó los modelos ARIMA (Autoregressive Intregrated Moving Average, siglas en inglés), planteada por Box-Jenkins (BJ) (1976), a partir de procesos auto regresivos y media móvil (MA, Moving Average, siglas en inglés). Este procedimiento permite especificar y estimar modelos para generar pronósticos de corto plazo con muestras representativas (grandes), (Fundamentos de Econometría Intermedia: Teoría y Aplicaciones, 2010, p. 183). En ese escenario, es objetivo lograr estimar el impacto de la ley 1696 sobre la demanda de alcohol en el caso de la industrial de Licores del Valle (ILV). La información con la que se trabaja es una serie de tiempo de las ventas mensuales en unidades de 750 cc., desde abril de 2008 hasta diciembre de 2014

Los modelos auto-regresivos se basan en la idea de que el valor actual de la serie, $\mathrm{Xt}$, puede explicarse en función de valores pasados Xt-1, Xt$2, X \mathrm{t}-3, \mathrm{Xt}-\mathrm{p}$, donde $\mathrm{p}$, determina el número de rezagos necesarios para pronosticar un valor actual, así un modelo AR (P) está determinado por:

$$
X_{t}=\varphi_{0}+\varphi_{1} X_{t-1}+\varphi_{2} X_{t-2}+\cdots+\varphi_{3} X_{t-p}+\varepsilon_{t}
$$

Donde $\varepsilon_{t}$ es un proceso ruido blanco.

Los Modelos de Media Móvil, (MA), suponen linealidad, el valor actual de la serie, $X_{t^{\prime}}$ está influenciado por los valores de la fuente externa y está determinado por:

$$
X_{t}=\varphi_{0}+\varphi_{1} \varepsilon_{t-1}+\varphi_{2} \varepsilon_{t-2}+\cdots+\varphi_{3} \varepsilon_{t-q}+\varepsilon_{t}
$$

Donde $\varepsilon_{t}$ es un proceso ruido blanco.

Aunque, es muy probable que una serie de tiempo, $X_{t}$, tenga características de AR y de MA a la vez $y$, por consiguiente, sea un ARMA. Así, sigue un proceso ARMA (p, q), en el que habrá términos $p$ auto-regresivos y q términos de media móvil.

$$
X_{t}=\varphi_{0}+\varphi_{1} X_{t-1}+\varphi_{2} X_{t-2}+\cdots+\varphi_{3} X_{t-p}+\varphi_{1} \varepsilon_{t-1}+\varphi_{2} \varepsilon_{t-2}+\cdots+\varphi_{3} \varepsilon_{t-q}+\varepsilon_{t}
$$

Donde $\varepsilon_{t}$ es un proceso ruido blanco. 
Para un proceso una condición de estacionariedad es la misma que para un proceso AR (p), del mismo modo una condición de invertibilidad es la misma que para el proceso MA (q).

\section{Hallazgos}

La figura 1 indica que la serie presenta un fuerte componente estacional sin tendencia en los meses de diciembre, por ser el mes de mayores fiestas y, por ende, de mayor consumo en la ciudad. Esto implica que la serie debe ser ajustada estacionalmente para poder trabajar con ella, ya que pueden invalidarse los resultados desde las pruebas de estacionariedad.

Siguiendo los elementos metodológicos del Instituto Nacional de Estadísticas INE (2008, p. 6): "El ajuste estacional se realizó con base en X12- ARIMA, el cual es un método basado en promedios móviles, los cuales se sustentan en el dominio del tiempo o en el de frecuencias y logra el ajuste estacional con el desarrollo de un sistema de los factores que explican la variación estacional en una serie. Este es un programa de código abierto, desarrollado por la oficina del censo de los Estados Unidos (U.S. Census Bureau 2000) a partir de los programas de ajuste estacional Census X-11 (Shishkin, 1967) (ZNG, 2008) de la oficina del censo de los Estados Unidos, y X11 ARIMA (Dagum 1980, 1988) de la oficina de estadística de Canadá. En los últimos años, X12 ARIMA ha adquirido relevancia en vista de que entre sus innovaciones se encuentran procedimientos basados en modelos ARIMA para cada uno de los componentes de la serie de tiempo de interés. Específicamente, considera los modelos Reg ARIMA, los cuales son modelos de regresión cuyos errores siguen un proceso ARIMA. Esta rutina de preajuste trata a los valores extremos y efectos especiales con modelos del tipo ARIMA, en él se estima un modelo ARIMA estacional (SARIMA)".

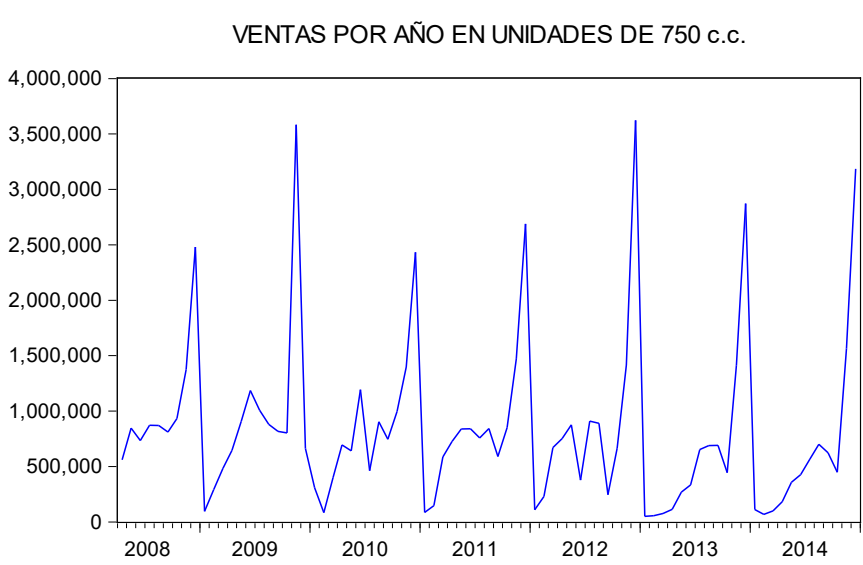

Figura 1. Ventas en Unidades de 750 CC periodo 2008-2014. Fuente: Elaborado por el autor a partir de datos de (ILV) Industria de Licores del Valle.

La figura 2 es la serie de tiempo Desestacionalizadas ${ }^{5}$ por medio del método de $\mathrm{X}-12$ census

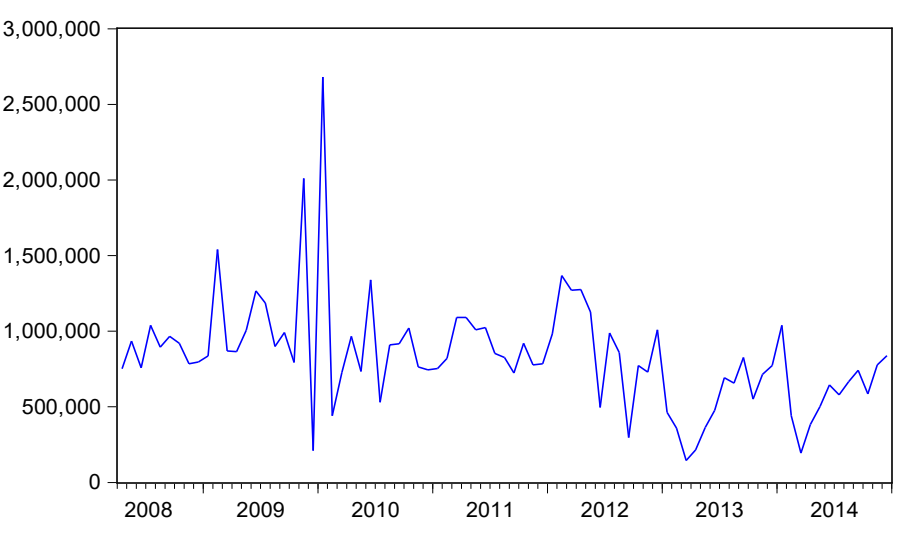

Figura 2. Ventas por año en unidades de 750 de C.C. Desestacionalizadas. Fuente: Elaborado por el autor a partir de datos de (ILV) Industria de Licores del Valle.

5 "La idea básica que sustenta la desestacionalización de las series económicas, es el hecho que a éstas las constituyen varias componentes no observables que pueden, en un momento dado, ser separadas de la serie original. Dichas componentes se utilizan para caracterizar los distintos movimientos que pueden presentar una serie, tales como la tendencia, el ciclo, la estacionalidad y el comportamiento irregular", (INE, 2008, p. 3). 
El correlograma indica que la serie puede ser modelada a partir de una estructura MA (2) (Guerrero, 2003, p. 127), lo cual indica que es estacionaria a nivel descontando por defecto el efecto de estacionalidad que se había establecido al principio en la figura 3.

\begin{tabular}{|c|c|c|c|c|c|c|}
\hline Autocorrelation & Partial Correlation & & $A C$ & PAC & Q-Stat & Prob \\
\hline 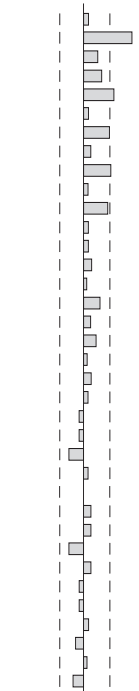 & 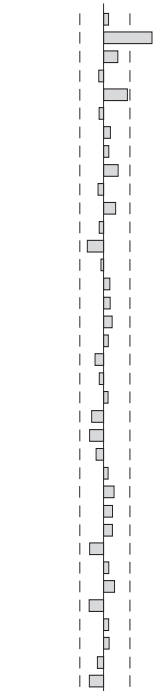 & $\begin{array}{r}1 \\
2 \\
3 \\
4 \\
5 \\
6 \\
7 \\
8 \\
9 \\
10 \\
11 \\
12 \\
13 \\
14 \\
15 \\
16 \\
17 \\
18 \\
19 \\
20 \\
21 \\
22 \\
23 \\
24 \\
25 \\
26 \\
27 \\
28 \\
30 \\
31 \\
32 \\
33 \\
34 \\
35 \\
36\end{array}$ & $\begin{array}{r}0.027 \\
0.432 \\
0.120 \\
0.157 \\
0.269 \\
0.037 \\
0.239 \\
0.051 \\
0.231 \\
0.039 \\
0.216 \\
0.030 \\
0.030 \\
0.062 \\
0.011 \\
0.137 \\
0.049 \\
0.112 \\
0.008 \\
0.053 \\
0.037 \\
-0.043 \\
-0.035 \\
-0.124 \\
-0.016 \\
-0.002 \\
0.044 \\
0.057 \\
-0.088 \\
0.066 \\
-0.034 \\
-0.040 \\
0.026 \\
-0.075 \\
0.023 \\
-0.091\end{array}$ & $\begin{array}{r}0.027 \\
0.432 \\
0.124 \\
-0.034 \\
0.212 \\
-0.025 \\
0.045 \\
0.029 \\
0.121 \\
-0.059 \\
0.105 \\
-0.040 \\
-0.143 \\
-0.020 \\
0.057 \\
0.048 \\
0.071 \\
0.036 \\
-0.073 \\
-0.047 \\
0.039 \\
-0.105 \\
-0.117 \\
-0.077 \\
0.038 \\
0.086 \\
0.076 \\
0.078 \\
-0.122 \\
0.035 \\
0.091 \\
-0.135 \\
0.029 \\
0.031 \\
-0.065 \\
-0.122\end{array}$ & $\begin{array}{l}0.0618 \\
15.967 \\
17.198 \\
19.355 \\
25.779 \\
25.902 \\
31.108 \\
31.351 \\
36.351 \\
36.491 \\
40.961 \\
41.051 \\
41.143 \\
41.530 \\
41.543 \\
43.484 \\
43.736 \\
45.072 \\
45.080 \\
45.389 \\
45.543 \\
45.755 \\
45.895 \\
47.710 \\
47.739 \\
47.740 \\
47.983 \\
48.399 \\
49.394 \\
49.970 \\
50.126 \\
50.354 \\
50.438 \\
51.232 \\
51.311 \\
52.560\end{array}$ & $\begin{array}{l}0.804 \\
0.000 \\
0.001 \\
0.001 \\
0.000 \\
0.000 \\
0.000 \\
0.000 \\
0.000 \\
0.000 \\
0.000 \\
0.000 \\
0.000 \\
0.000 \\
0.000 \\
0.000 \\
0.000 \\
0.000 \\
0.001 \\
0.001 \\
0.001 \\
0.002 \\
0.003 \\
0.003 \\
0.004 \\
0.006 \\
0.008 \\
0.010 \\
0.010 \\
0.012 \\
0.016 \\
0.021 \\
0.027 \\
0.029 \\
0.037 \\
0.037\end{array}$ \\
\hline
\end{tabular}

Figura 3. Correlograma de la serie.

Fuente: Elaboración propia con base en estadísticas de la ILV.

Los resultados indican que la serie carece de procesos de caminata aleatoria, es decir, no presenta raíz unitaria. Esto indica que la serie puede ser modelada a nivel con un MA (2), extraído del co-
Una vez realizado el correlograma se pasa a aplicar la prueba del ADF para la serie de tiempo de las ventas de licores Desestacionalizadas. Los resultados se muestran en la tabla 1.

Tabla 1. Prueba de ADF (1)

\section{Null Hypothesis: SALES_SA has a unit root}

Exogenous: Constant

Lag Length: 1 (Automatic - based on SIC, maxlag=11)

t-Statistic Prob.*

Augmented Dickey-Fuller test statistic $-3.826869$

0.0040

Test critical $1 \%$

values: level

$5 \%$

level

$-3.515536$

$10 \%$

$-2.898623$

level

$-2.586605$

*MacKinnon (1996) one-sided p-values.

\section{Fuente: cálculo de los autores.}

rrelograma, incluida la variable Dummy que indica la aplicación de la ley. El modelo que incorpora la Dummy $^{12}$ que especifica la aplicación de la ley 1689 de diciembre de 2013 es el siguiente, (ver tabla 2):

Tabla 2. Resultados del modelo con efectos de ley

\begin{tabular}{|c|c|c|c|c|}
\hline Variable & Coefficient & Std. Error & t-Statistic & Prob. \\
\hline $\mathbf{C}$ & 834304.4 & $\mathbf{5 2 3 2 7 . 5 9}$ & 15.94387 & 0.0000 \\
\hline MA(2) & 0.383789 & 0.104051 & 3.688471 & 0.0004 \\
\hline R-squared & 0.165306 & \multicolumn{2}{|c|}{ Mean dependent var } & 834222.8 \\
\hline Adjusted R-squared & 0.154740 & \multicolumn{2}{|c|}{ S.D. dependent var } & 372040.9 \\
\hline S.E. of regression & 342047.0 & \multicolumn{2}{|c|}{ Akaike info criterion } & 28.34767 \\
\hline Sum squared resid & $9.24 E+12$ & \multicolumn{2}{|c|}{ Schwarz criterion } & 28.40679 \\
\hline Log likelihood & -1146.080 & \multicolumn{2}{|c|}{ Hannan-Quinn criter. } & 28.37139 \\
\hline F-statistic & 15.64547 & \multicolumn{2}{|c|}{ Durbin-Watson stat } & $\mathbf{1 . 9 8 0 2 0 6}$ \\
\hline Prob(F-statistic) & 0.000166 & & & \\
\hline
\end{tabular}


Como se puede ver en la tabla 2, la variable Dummy no es significativa al interior del modelo. Esto implicaría inicialmente que la ley no ha presentado efectos sobre la venta de licor. Al realizar el ajuste, el modelo final estimado es el siguiente, (ver tabla 3):

Tabla 3. Modelo final

\begin{tabular}{lllll}
\hline Variable & Coefficient & Std. Error & t-Statistic & Prob. \\
& & & & \\
C & 834304.4 & 52327.59 & 15.94387 & 0.0000 \\
MA(2) & 0.383789 & 0.104051 & 3.688471 & 0.0004 \\
R-squared & $\mathbf{0 . 1 6 5 3 0 6}$ & Mean dependent var & 834222.8 & 0.0014 \\
Adjusted R-squared & $\mathbf{0 . 1 5 4 7 4 0}$ & S.D. dependent var & 372040.9 & 28.40679 \\
S.E. of regression & 342047.0 & Akaike info criterion & 28.34767 & 28.37139 \\
Sum squared resid & $\mathbf{9 . 2 4 E + 1 2}$ & Schwarz criterion & & 1.980206 \\
Log likelihood & $\mathbf{- 1 1 4 6 . 0 8 0}$ & Hannan-Quinn criter. & & \\
F-statistic & $\mathbf{1 5 . 6 4 5 4 7}$ & Durbin-Watson stat & & \\
Prob(F-statistic) & $\mathbf{0 . 0 0 0 1 6 6}$ & & & \\
\end{tabular}

Fuente: Cálculo de los autores.

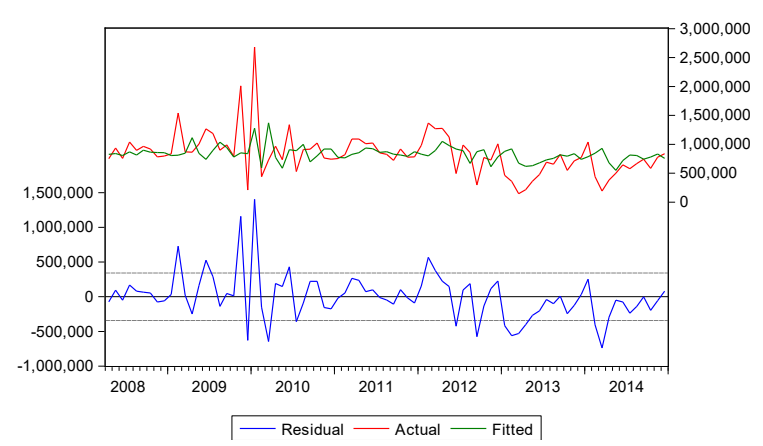

Figura 4. Modelo MA (2) de la serie de estudio Fuente: Cálculo de los autores.

Al interior del análisis econométrico se evidenció que la ley, efectivamente, no ha tenido efecto sobre la cantidad vendida de licor. Esto indica que, para efectos del presente trabajo, se valida la hipótesis sobre la no significancia de la ley. Lo anterior no implica que dicha ley no esté funcionando.

Debido al alcance del estudio resulta pertinente realizar estudios más detallados sobre el impacto de esta ley en los accidentes de tránsito debido a altos grados de alicoramiento. Con- viene también incluir un efecto de interacción entre la ley y las fotomultas que tiene el municipio, así como el impacto de cada uno de los artículos de la ley.

Se puede tener un marco de ampliación del estudio en una línea relacionada con los análisis de intervención en el tiempo sobre este tipo de medida y realizar un análisis de mediano y largo plazo para el mismo, en aras de saber si efectivamente tendrá un impacto sobre las ventas de licores.

\section{Conclusiones}

Las estadísticas sobre consumo de bebidas alcohólicas a nivel del continente, muestran que Colombia ocupa el cuarto puesto. En los últimos 10 años el crecimiento en el consumo de bebidas alcohólicas ha estado en niveles del 7.3\% anual, siendo la cerveza el producto líder y representa no menos del $80 \%$. En términos de alcohol, el nivel de consumo se ha mantenido cercano al $2.5 \%$ anual en dicho periodo, destacando que los licores importa- 
dos han ido ganando participación en ese mercado.

Las bebidas alcohólicas son muy importantes en términos tributarios para los departamentos, los recaudos por impuestos al alcohol y la cerveza son cerca del $60 \%$, no menos de $\$ 3.5$ billones se recaudan por año por ese concepto en los departamentos. En el Valle del Cauca, en el año 2001, se recaudaron \$236 miles de millones y en 2009 la cifra alcanzó \$353.6 miles de millones, para un crecimiento del $5.1 \%$ anual. En Colombia, ese crecimiento fue del $4.25 \%$ anual en el mismo periodo. En ese orden, una caída en el consumo pondría en riesgo las finanzas departamentales, dado el alto nivel de dependencia que se observa del impuesto a los licores.

Hay, por tanto, una ambivalencia. Por un lado, la legislación es cada vez más "dura" en términos de la regulación sobre la venta, el consumo y los efectos nocivos que las bebidas alcohólicas tienen para el individuo y la sociedad; pero, de otra parte, se defiende el monopolio departamental para la venta de alcohol y de esa forma mantener el nivel de ingresos fiscales en los departamentos.

Estudios preliminares muestran que la demanda de bebidas alcohólicas, a pesar de la regulación del estado, es sumamente compleja y responde a estructuras muy definidas de estrategias que involucran consumidores, competidores, innovación, publicidad, eventos, estratos, géneros, ingresos y precios. En general, la demanda es muy peleada por cada uno de los productos en el mercado, puesto que la industria de bebidas alcohólicas es de las dinámicas e innovadoras en el mercado. Bajo ese argumento, se procuró establecer si había efectos regresivos sobre la demanda dado un elemento legal como lo es la Ley 1696 de 2013.

Bajo un modelo econométrico tipo ARIMA, se muestra que evidentemente no hay un efecto de la Ley 1696 (2013) sobre la demanda de alcohol (botella de 750cc), al menos en el caso del Valle del Cauca. Lo que da a comprender que son, efecti- vamente, otras variables como los gustos, las preferencias, los competidores, aquellos que puedan tener una incidencia sobre la demanda de este tipo de productos.

De otra parte, se infiere de forma indirecta que no hay relación marcada entre el consumo de bebidas alcohólicas y los que conducen, si bien es cierto que existen casos, eso no significa que existe correlación entre el consumo y la demanda de alcohol y los agentes que conducen y tienen vehículos. Es posible que se pueda, en un estudio posterior, lograr observar de forma más detallada si hay o no un efecto, y para ello es importante tener una observación de más largo plazo.

Finalmente, el estudio es significativo en la medida en que logra mostrar el efecto de la Ley 1696 (2013) sobre la demanda de alcohol y deja un insumo para que se pueda indagar en estudios sub-siguientes temas que le aportarían mucho a este sector. Como, por ejemplo, ahondar en los procesos de medición de elasticidad de precios y de ingresos, elasticidades de sustitución y el impacto que estos puedan tener no solo en estrategias de mercado para los productos de los monopolios departamentales, sino el impacto que ello pueda llevar sobre las finanzas departamentales.

\section{Referencias bibliográficas}

Afanador, J. y Joya, M. (2013). Oportunidades y Retos para Diageo con la firma del TLC Colombia - Unión Europea. Universidad Colegio mayor del Rosario (tésis inédita de pregrado). Universidad del RosarioBogotá, Colombia.

Cante, F. (2007). Acción Colectiva, Metapreferencias y Emociones. Cuadernos de Economía. XXVI, intervalo de páginas. Recuperado de http://www.revistas.unal.edu.co/index.php/ceconomia/article/ view/1081/1590

Decreto No. 120 (21 de enero de 2010), por el cual se expide normatividad sobre el consumo de alcohol. Diario oficial $n .{ }^{\circ} 47599$ 
Decreto No. 1355 (04 de agosto de 1970), por el cual se dictan normas sobre policía Diario oficial $n .^{\circ} N D$

Giraldo, Norman (2006). Notas de clase sobre series de tiempo. Inédito. Centro de documentación Rafael Botero. Universidad Nacional de Colombia, Medellín.

Guerrero, V. M. (2003). Análisis estadistico de series de tiempo económicas (Segunda ed.). Ciudad de México: Thomson.

ILV (2014). Industria licorera del Valle, Ron Marqués del Valle Cinco Años. Recuperado de http://www.ilvalle.com.co/

ILV (2014). Industria licorera del Valle, Aguardiente Blanco del Valle sin Azúcar. Recuperado de http://www. ilvalle.com.co/

INE. (Septiembre de 2008). Desestacionalización - X12 - ARIMA - con efecto calendario Índice Supermercados. Recuperado de http://www.ine.cl/canales/ menu/publicaciones/estudios_y_documentos/estudios/estudioscnportadas/n12.pdf

Lavoie, M (2005). La economía post-keynesiana. Icária Editorial. ISBN 84-7426-785-4. Barcelona España. Recuperado de https://books.google.com.co/books?i$\mathrm{d}=4$ AZr8_Jgk_MC\&pg = PA39\&lpg = PA39\&d$\mathrm{q}=$ lancaster+el+principio+de+separacion\&sour$c e=b 1 \&$ ots $=7 v Q-3 u E y f Q \&$ sig $=$ gwIyDj9QGPAjpLr1uM7RyjhEGtI\&hl=es\&sa=X\&ei=wh-UVef6MIPq-QHDvJa4Aw\&ved = 0CCEQ6AEwAQ\# $\mathrm{v}=$ onepage $\& \mathrm{q}=$ lancaster $\% 20 \mathrm{el} \% 20$ principio $\% 20$ $\mathrm{de} \% 20$ separacion $\& \mathrm{f}=$ false

Ley No. 30 Reglamentada por el Decreto Nacional 3788 de 1986 (31 de enero), por la cual se adopta el estatuto nacional de estupefacientes y se dictan otras disposiciones Diario oficial $n .^{\circ} \mathrm{ND}$

Ley No. 1696 (19 de diciembre de 2013), por medio de la cual se dictan disposiciones penales y administrativas para sancionar la conducción bajo el influjo del alcohol u otras sustancias psicoactivas. Diario oficial No. 49009 .

Organización Mundial de la Salud OMS (2002). Informe sobre la salud del mundo 2002. Reducir los Riesgos y
Promover una Vida Sana. Recuperado de http://www. who.int/whr/2002/es/

Manquillo, C. , Mora, D., y Sánchez, Y.. (2012). Planeamiento Estratégico de la Industria de. Centro de negocios de la Pontificia Universidad Católica del Perú, 110.

Martínez, J. (2004). Tipos de Elección Racional. Revista internacional de Sociología. Universidad de la laguna. Santa Cruz de Tenerife. Recuperado de http://josamaga. webs.ull.es/Papers/tipos-rat.pdf

Minsalud Colombia. (2013). Estudio Nacional sobre Consumo de Sustancias Psicoactivas en Colombia. Estudio realizado por el Gobierno Nacional de la República de Colombia, a través del Ministerio de Justicia y del Derecho - Recuperado de www.odc.gov.co

Rehm, et al (2009). Global burden of disease and injury and economic cost attributable to alcohol use and alcohol-use disorders. The Lancet. 373., 2223-2233. Recuperado de http://www.sciencedirect.com/science/article/ pii/S0140673609607467\#

Sierra, Freddy Alexander (2011) La legislación de la lucha antialcohólica en Santander (1923-1928), Anuario de Historia Regional y de las Fronteras, 16, intervalo de páginas.

Tenjo, J. (2013) Opinión Políticas de mercado que pueden ayudar a controlar el alcoholismo. Recuperado de http://www.utadeo.edu.co/es/noticia/opinion/economia/63/politicas-de-mercado-que-pueden-ayudar-controlar-el-alcoholismo

Zapata, et al (2012). Una estimación de la adulteración y de la falsificación de bebidas alcohólicas en Colombia. La imprenta editores S.A Bogotá. Recuperado de Repository.fedesarrollo.org.co/handle/11445/2908

Zuleta, LA. (2000) Fedesarrollo La industria de licores en Colombia. Recuperado de http://www.fedesarrollo.org.co/wp-content/uploads/2011/08/ La-industria-de-licores-en-colombia-TEXTO-FINAL.pdf 